\title{
Near-infrared light-triggered theranostics for tumor-specific enhanced multimodal imaging and photothermal therapy
}

This article was published in the following Dove Press journal:

International Journal of Nanomedicine

16 June 2017

Number of times this article has been viewed

\author{
Bo $\mathrm{Wu}^{1, *}$ \\ Bing Wan ${ }^{2, *}$ \\ Shu-Ting Lu' \\ Kai Deng ${ }^{3}$ \\ Xiao-Qi $\mathrm{Li}^{\prime}$ \\ Bao-Lin Wu' \\ Yu-Shuang $\mathrm{Li}^{\prime}$ \\ Ru-Fang Liao' \\ Shi-Wen Huang ${ }^{3}$ \\ Hai-Bo $X u^{1,2}$ \\ 'Department of Radiology, Zhongnan \\ Hospital of Wuhan University, \\ ${ }^{2}$ Department of Radiology, Union \\ Hospital of Tongji Medical College, \\ Huazhong University of Science \\ and Technology, ${ }^{3}$ Department of \\ Chemistry, Key Laboratory of \\ Biomedical Polymers, Ministry of \\ Education, Wuhan University, Wuhan, \\ People's Republic of China \\ *These authors contributed equally \\ to this work
}

Correspondence: Shi-Wen Huang Department of Chemistry, Key Laboratory of Biomedical Polymers, Ministry of Education, Wuhan University, 299 Bayi Road, Wuhan 430072,

People's Republic of China

Tel +86276875 5317

Email swhuang@whu.edu.cn

Hai-Bo Xu

Department of Radiology, Zhongnan

Hospital of Wuhan University,

169 Donghu Road, Wuhan,

People's Republic of China

Tel +8627 678I 2533

Email xuhaibol I20@hotmail.com
Abstract: The major challenge in current clinic contrast agents (CAs) and chemotherapy is the poor tumor selectivity and response. Based on the self-quench property of IR820 at high concentrations, and different contrast effect ability of Gd-DOTA between inner and outer of liposome, we developed "bomb-like" light-triggered CAs (LTCAs) for enhanced CT/MRI/ FI multimodal imaging, which can improve the signal-to-noise ratio of tumor tissue specifically. IR820, Iohexol and Gd-chelates were firstly encapsulated into the thermal-sensitive nanocarrier with a high concentration. This will result in protection and fluorescence quenching. Then, the release of CAs was triggered by near-infrared (NIR) light laser irradiation, which will lead to fluorescence and MRI activation and enable imaging of inflammation. In vitro and in vivo experiments demonstrated that LTCAs with $808 \mathrm{~nm}$ laser irradiation have shorter $\mathrm{T}_{1}$ relaxation time in MRI and stronger intensity in FI compared to those without irradiation. Additionally, due to the high photothermal conversion efficiency of IR820, the injection of LTCAs was demonstrated to completely inhibit C6 tumor growth in nude mice up to 17 days after NIR laser irradiation. The results indicate that the LTCAs can serve as a promising platform for NIR-activated multimodal imaging and photothermal therapy.

Keywords: light triggered, near-infrared light, tumor-specific, multimodal imaging, photothermal therapy, contrast agents

\section{Introduction}

Early diagnosis and proper disease management of tumor, as well as the personalized medicine of tumor, depend on the precise diagnosis of pathological states of tumor. Various noninvasive imaging techniques such as computed X-ray tomography (CT)/ magnetic resonance imaging (MRI)/fluorescence imaging (FI) have been widely applied in diagnosis of tumor in clinical practice. ${ }^{1,2}$ Since CT, MRI, and optical imaging all have their own strengths and weaknesses, the combination of these imaging techniques is more preferable for comprehensive and precise information of the tumor.

The CT/MRI imaging signal can be modulated with contrast agents (CAs) by absorbing X-ray or shortening the relaxation time of water protons to improve the visibility. However, the intrinsic limitation about poor soft tissue resolutions, radiation, and renal toxicity of commercial CAs have limited commercial CAs clinical application. ${ }^{3,4}$ In order to improve the modest signal/noise ratio of cancerous tissues, lots of carriers, which are responsive to such external stimuli as $\mathrm{pH}^{, 5-7}$ redox, ${ }^{8}$ temperature,${ }^{9}$ enzyme,${ }^{10}$ and competitive binding, ${ }^{11}$ have been developed. Nevertheless, acquiring tumor imaging specifically, spatially and temporally has been still a big challenge for CAs. 
Recently, light-induced release systems of drugs offering on-demand targeting capability have provided a new unique opportunity for image-guided therapies. ${ }^{12}$ The photosensitive capped delivery systems have attracted much attention owing to their remarkable therapeutic efficacy. Using light to generate heat has been proven to improve specific release and cellular uptake of theranostics agents. ${ }^{13}$ Upon near-infrared (NIR) light laser irradiation, the dissipation of absorbed light energy as heat can not only mediate hyperthermia of tumor with high spatial and temporal resolution but also activate the rapid release of contents at the site of interest. ${ }^{14-17}$ The introducing of the light-triggered release systems in CA delivery will help to utilize the self-quench property of fluorescence at high concentrations, and has been used for optic imaging. ${ }^{18}$ However, previous reports on light-triggered nanotheranostics mostly focused on cancer treatment. So far, few reports have used light-triggered nanotheranostics to improve the imaging effect of clinical use CAs. Because of that, Gd-DOTA has different contrast effect when it was inner and outer of liposome, ${ }^{19}$ light-triggered nanotheranostics will be one of the most promising delivery to improve the signal-to-noise ratio of tumor tissue specifically.

Photothermal therapy (PTT) is based on photosensitive agents aimed at localized destruction of cancer cells by conversion of NIR light into heat. ${ }^{20}$ To date, various nanosized photothermal agents have been developed for precise localization and intensity control of heating, which effectively improves the hyperthermia ablation therapy. ${ }^{21,22}$ However, the most current photothermal agents used in the NIR range are inorganic nanomaterials that are not easily excreted in biological environment. Thus, it is necessary to develop NIR-absorbing organic nanomaterials to reduce the long-term risks of PTT. IR820 (new indocyanine green) is a sort of cyanine dye, which has more stability under different temperatures, longer tissue retention time, and stronger fluorescent signals. ${ }^{23}$ IR820 generates sufficient heat upon NIR laser irradiation to create a cytotoxic effect, which is similar to indocyanine green, but superior to indocyanine green in terms of stability. IR820 can be easily metabolized by the liver. ${ }^{24,25}$ Due to its good biosecurity, IR820 was employed as the photothermal agent in this study. ${ }^{26,27}$

To further improve the theranostic efficiency and provide a way to image the pathological characteristics of tumors, it is necessary to develop a more effective theranostic agent to sense subtle pathological changes. The lipid-polymer hybrid nanoparticles, which have various advantages of polymeric nanoparticles and liposomes, have become a robust delivery platform with properties such as excellent stability, tunability, high drug encapsulation, and sustained drug release. ${ }^{28}$ Liposome was an ideal CAs carrier to clearly display the extent of cancer and monitors the treatment effect in real time. ${ }^{29,30}$ In addition, previous research has suggested that CAs have better MRI contrast effect or stronger fluorescence intensity when the CAs were released from the nanoparticles. ${ }^{31}$

In this account, a novel strategy by using an NIR laser to turn on CT/MRI/FI multimodal imaging and PTT was successfully developed, based on the self-quench property of IR820 at high concentrations and different contrast effect ability of Gd-DOTA in the inner and outer parts of liposomes. IR820 was loaded into the core of polyethylene glycol (PEG)protected dipalmitoylphosphatidylcholine (DPPC)/distearoyl phosphatidylcholine (DSPC) thermosensitive liposomes. In addition to the photothermal agent IR820, clinically widely used CA Gd-chelates (Magnevist) for MRI and iodine compound (Iohexol) for CT were also stored in the core of light-triggered CAs (LTCAs), which could result in protection and FI quenching in the systemic circulation. As another important element, $\mathrm{NH}_{4} \mathrm{HCO}_{3}$, which at a high temperature $\left(40^{\circ} \mathrm{C}-42^{\circ} \mathrm{C}\right)$ can decompose to $\mathrm{CO}_{2}$ bubbles, was also incorporated into the aqueous compartment of liposomes. The multimodal imaging could be turned on by the release of CAs triggered by NIR laser. This simple but powerful strategy for responsive tumor-specific imaging will be very significant for tumor-specific diagnosis and treatment.

\section{Experimental section}

\section{Materials}

DPPC, DSPC, and 1,2-distearoyl-sn-glycero-3-phosphoethanolamine-N-[methoxy(polyethylene glycol)] (DSPEPEG) was purchased from Shanghai Advanced Vehicle Technology Co., Ltd. (Shanghai, People's Republic of China). Magnevist was purchased from Yangtze River Pharmaceutical Group (Nanjing, People's Republic of China). Iohexol was purchased from GE Healthcare (Dublin, Ireland). All the chemicals were of analytical grade and used as received without further purification. C6 cell line was purchased from the China Center for Type Culture Collection (Wuhan University). The consumables of cell culture in this work were purchased from Beijing Dingguo Changsheng Biotechnology Co., Ltd (Beijing, People's Republic of China).

\section{Synthesis of LTCA liposomes}

The LTCAs were prepared by a matrix-encapsulation method. ${ }^{32}$ DPPC (33 mg), DSPC (12 mg), and DSPE-PEG $(16.2 \mathrm{mg}$ ) were dissolved thoroughly in $8 \mathrm{~mL}$ methanol under vigorous stirring. Then, the mixture was added to $12 \mathrm{~mL}$ 
ultrapure water solution containing Magnevist $(460 \mu \mathrm{L})$, Iohexol (105 $\mu \mathrm{L})$, IR820 (25 mg), and $\mathrm{NH}_{4} \mathrm{HCO}_{3}(0.95 \mathrm{mg})$ under stirring. The mixture was then stirred at room temperature for $12 \mathrm{~h}$ to remove the methanol by volatilization. After that, the solution was processed with sequential extrusions through 800, 400, 200, and $100 \mathrm{~nm}$ polycarbonate filters (six times each) to remove any possible remnants. Finally, the liposome was concentrated (3,500 r/min) using an amicon Ultra-4 centrifugal filter (Millipore, Billerica, MA, USA; molecular weight cutoff $[\mathrm{MWCO}]=8,000)$.

\section{Characterization}

The size and size distribution of LTCAs were characterized by dynamic light scattering (DLS; Nano-ZS ZEN3600, Malvern Instruments, Malvern, $\mathrm{UK}$ ) at $25^{\circ} \mathrm{C}$. After negatively staining with phosphotungstic acid, transmission electron microscopy (TEM; JEM-2100 microscope) was used to observe the morphology and structure of LTCAs. To measure the loading efficiency and drug encapsulation efficiency, a predetermined aliquot of LTCAs was collected by freeze-drying, and then the dry nanoparticles were dissolved in ultrapure water. After that, the IR820 concentration in ultrapure water was measured by fluorescence measurement using a calibration curve constructed from IR820 solutions with different concentrations. The concentrations of Gd(III) were measured by inductively coupled plasma mass spectrometry (ICP-AES; IRIS Intrepid II XSP, Thermo Elemental [Waltham, MA, USA]). $\mathrm{I}^{-}$was measured using the chloric acid method as in a previously reported study. ${ }^{33}$

\section{LTCA release}

The release profile was studied using the dialysis method. In brief, $0.5 \mathrm{~mL}$ of LTCAs was transferred into a dialysis tube (MWCO =3,500) after NIR laser irradiation for $10 \mathrm{~min}$ $\left(808 \mathrm{~nm}, 1 \mathrm{~W} / \mathrm{cm}^{2}\right)$ as in the aforementioned photothermal procedure. Then, the dialysis tube was immersed into a $50 \mathrm{~mL}$ tube containing $20 \mathrm{~mL}$ dextrose solution (fraction 5\%) in a shaker at $37^{\circ} \mathrm{C}$ and $120 \mathrm{rpm}$. At predetermined times $(5,10$, $15,20,30,45$, and $60 \mathrm{~min}$ ), $5 \mathrm{~mL}$ of the external dextrose solution was replaced with the corresponding fresh dextrose solution. The concentration of $\mathrm{Gd}$ (III) was determined by ICP-AES, and $\mathrm{I}^{-}$was measured using the chloric acid method. IR820 quantity was measured by fluorescence measurement $(\mathrm{Ex}=785 \mathrm{~nm}, \mathrm{Em}=830 \mathrm{~nm})$.

\section{Photothermal profile}

The LTCAs with various concentrations of IR $820(0,0.5,1,2$, 4 , and $8 \mu \mathrm{M}$ ) were transferred into a quartz cuvette. In order to avoid the possible solvent evaporation and increase of LTCA concentration caused by the photothermal heating, the quartz cuvette was capped and wrapped with parafilm and then irradiated by $1 \mathrm{~W} / \mathrm{cm}^{2}$ NIR laser for $10 \mathrm{~min}$. In addition, the LTCA solution (IR820: $8 \mu \mathrm{M}$ ) was illuminated with various power densities of NIR laser for $10 \mathrm{~min}$. The temperature of the LTCA solution was recorded using a noncontact infrared thermal imager to ensure the accuracy of the measurement. Equal volume of PBS was used as the experimental control.

\section{In vitro photothermal cytotoxicity study}

C6 glioma-astrocytoma cell lines (C6 cells) were maintained in high-glucose DMEM medium (Gibco-BRL, Suzhou, People's Republic of China) containing 10\% fetal bovine serum (Biological Industries Inc., Beit Haemek, Israel) and 1\% penicillin-streptomycin (Gibco-BRL) in a humidified atmosphere of $5 \% \mathrm{CO}_{2}$ at $37^{\circ} \mathrm{C}$. The photothermal cytotoxicity assessment of LTCAs was determined by tetrazolium-based colorimetric assay (MTT test). Firstly, C6 cells $\left(5 \times 10^{3}\right)$ were seeded into 96 -well plates for $12 \mathrm{~h}$ to allow cell attachment. The cells were then incubated with different concentrations of LTCAs (IR820: 0, 0.5, 1, 2, 4, and $8 \mu \mathrm{M}$ ) for $12 \mathrm{~h}$. Then, the medium was removed and illuminated with NIR laser $\left(1 \mathrm{~W} / \mathrm{cm}^{2}, 15 \mathrm{~min}\right)$. After that, $20 \mu \mathrm{L}$ of MTT solution $(5.0 \mathrm{mg} / \mathrm{mL})$ was added to each well and incubated for a further $4 \mathrm{~h}$ at $37^{\circ} \mathrm{C}$. Then, the medium was removed, and $200 \mu \mathrm{L}$ of dimethyl sulfoxide was added to each well. The optical density of samples at $570 \mathrm{~nm}$ was recorded using a plate reader. The relative cell viability was the ratio of treatment groups to control. The effect of NIR laser power density $\left(0,0.5,0.8,1.0\right.$, and $\left.1.2 \mathrm{~W} / \mathrm{cm}^{2}\right)$ on cell viability was also evaluated as in the aforementioned photothermal procedure, and the IR 820 concentration was fixed at $8 \mu \mathrm{M}$.

\section{In vitro light-triggered $C T$ and MRI imaging}

Firstly, the LTCA solution was placed in a quartz cuvette and irradiated by NIR laser for $10 \mathrm{~min}$ at $1 \mathrm{~W} / \mathrm{cm}^{2}$. C6 cells were seeded in $25 \mathrm{~cm}^{2}$ flask and allowed to grow to $\sim 80 \%$ density. Then, the medium was replaced by $2 \mathrm{~mL}$ of fresh medium containing various concentrations of LTCAs $\left(\mathrm{I}^{-}: 2,4,6,8\right.$, and $10 \mu \mathrm{M})$. After incubation with LTCAs for $4 \mathrm{~h}$, the medium was replaced by $1.5 \mathrm{~mL}$ of cell lysis reagent. The cell lysis solution was then collected for CT imaging. CT scans were performed using a GE Light Speed VCT imaging system at $80 \mathrm{kV}$ and $100 \mathrm{~mA}$, with a slice thickness of $0.625 \mathrm{~mm}$. As a control group, the LTCA solution without irradiation was also evaluated as in the aforementioned procedure. In vitro 
light-triggered MRI imaging was determined by 3.0-T clinical MRI scanner system (Siemens, Berlin, Germany) at T1WI and T1 map sequences with TR $15 \mathrm{~ms}$ and TE 2.1 ms. The cell sample was prepared as in the aforementioned CT procedure; the incubated concentrations of Gd(III) were $0.06,0.12,0.18,0.24$, and $0.3 \mathrm{mM}$.

\section{Animals}

Female BALB/c nude mice were purchased from Beijing HFK Bioscience Co. Ltd. with body weights of 16-18 g and housed under the standard pathogen-free conditions ( $60 \%$ relative humidity, $25^{\circ} \mathrm{C}$ room temperature). Mice were housed for 3 days prior to treatment. All mice were subcutaneously inoculated with $\mathrm{C} 6$ cells $\left(2 \times 10^{6}\right.$ per mice $)$ at backside to establish the subcutaneous transplanted glioma model. All procedures in this experiment were compliant with the local animal ethics committee. Procedures involving animals and their care were conducted in conformity with EU Directive 2010/63/EU for animal experiments and were approved by the Animal Welfare Committee of the Animal Experiment Center of Wuhan University.

\section{In vivo $C T / M R I / F I$ imaging}

C6 tumor-bearing $\mathrm{BALB} / \mathrm{c}$ nude mice were injected with the LTCA solution $(200 \mu \mathrm{L})$ through the tail vein. After the injection, the tumor CT images of mice with or without laser irradiation were obtained with GE Light Speed VCT imaging system (80 KV, $100 \mathrm{~mA}$, slice thickness $0.625 \mathrm{~mm})$. The in vivo MRI imaging was also evaluated as in the aforementioned procedure and performed on a $3.0 \mathrm{~T}$ clinical MRI scanner system (Siemens) at T1WI, T1 map sequences with TR $15 \mathrm{~ms}$ and TE $2.1 \mathrm{~ms}$. FI imaging was conducted with a PerkinElmer IVIS system (PerkinElmer, Inc., Santa Clara, USA). Tumor imaging before irradiation was used as control.

\section{In vivo photothermal imaging}

For in vivo photothermal study, when tumor volume grew to $450 \mathrm{~mm}^{3}$ (about 7 days after inoculation), the LTCA solution $($ dose $=8 \mathrm{mg}[\mathrm{IR} 820] / \mathrm{kg}, 200 \mu \mathrm{L})$ or PBS $(200 \mu \mathrm{L})$ was injected to $\mathrm{C} 6$ tumor-bearing $\mathrm{BALB} / \mathrm{c}$ nude mice through the tail vein. One and a half hours after the injection, the tumor site was irradiated by $808 \mathrm{~nm}$ NIR laser for $20 \mathrm{~min}$, and the temperature and photothermal imaging were recorded by an infrared thermal imaging system at different time intervals $(0,0.5,1,2,5,10$, and $20 \mathrm{~min})$.

\section{Antitumor assessment and histological analysis}

When tumor volume grew to around $200 \mathrm{~mm}^{3}$ (about 5 days after inoculation), the mice were divided into four groups (four mice in each group): (a) PBS, (b) laser irradiation only, (c) LTCAs without laser irradiation (dose $=10 \mathrm{mg} / \mathrm{kg}, 200 \mu \mathrm{L}$ ), and (d) LTCAs with laser irradiation (dose $=10 \mathrm{mg} / \mathrm{kg}, 200 \mu \mathrm{L})$. Then, the groups $\mathrm{b}$ and $\mathrm{d}$ were exposed to $808 \mathrm{~nm}$ laser $\left(1 \mathrm{~W} / \mathrm{cm}^{2}, 15 \mathrm{~min}\right) 1.5 \mathrm{~h}$ after the injection. Tumor volumes and mice weights were recorded. The tumor volume was calculated according to the following formula: $\mathrm{V}=\mathrm{ab} / 2$. $\mathrm{V}$ is the volume of the tumor and $a$ and $b$ are the tumor length and width, respectively. The mice were sacrificed at 17 days, and the tumors were dissected for hematoxylin-eosin (H-E) staining and evaluation of antitumor efficacy. Average weights of tumors at the end of photothermal treatment were calculated by analysis of variance. Statistical analyses were performed using PASW Statistics (version 17.0) software.

\section{Results and discussion Synthesis and characterization of liposome LTCAs}

In order to realize the responsive enhancement imaging of the tumor, we firstly prepared the light-triggered nanocarrier system LTCAs. The LTCAs were fabricated via matrixencapsulation method. ${ }^{32}$ Firstly, DPPC, DSPC, and DSPEPEG were dissolved in $8 \mathrm{~mL}$ methanol, and the mixture was dripped slowly into water solution, followed by sonication using a microtip probe sonicator $(60 \mathrm{~W})$. Afterwards, the mixture was then stirred for evaporation of methanol. As shown in Figure 1, the LTCAs comprised three components: a hydrophilic core, a thermoresponsive phospholipid bilayer (DPPC/DSPC), and a hydrophilic PEG shell. As expected, Iohexol, Magnevist, and IR820 were successfully entrapped into the core of LTCAs. $\mathrm{NH}_{4} \mathrm{HCO}_{3}$, which could generate $\mathrm{CO}_{2}$ bubbles when illumined by NIR laser, was also encapsulated for creating permeable defects in the lipid membrane. TEM imaging showed that LTCAs could be well dispersed in aqueous solution with a clear core-shell structure and smooth surface (Figure 2A). DLS results showed that the as-prepared LTCAs have good monodispersity with polydispersity index below 0.350 and average size between 104 and $135 \mathrm{~nm}$ (Figure 2B), which was conducive to CA accumulation in tumor tissue through the enhanced permeability and retention effect. Furthermore, efficient encapsulation of the loading into the aqueous phase of formulation is shown in Table 1.

In order to examine the light-triggered disassembly performance of LTCAs, the size change of LTCAs in response to $808 \mathrm{~nm}$ laser irradiation in PBS was monitored by DLS. DLS measurements showed that after laser irradiation for $10 \mathrm{~min}\left(1 \mathrm{~W} / \mathrm{cm}^{2}\right)$, the size distribution and monodispersity of LTCAs were significantly increased (Figure 2B), which 


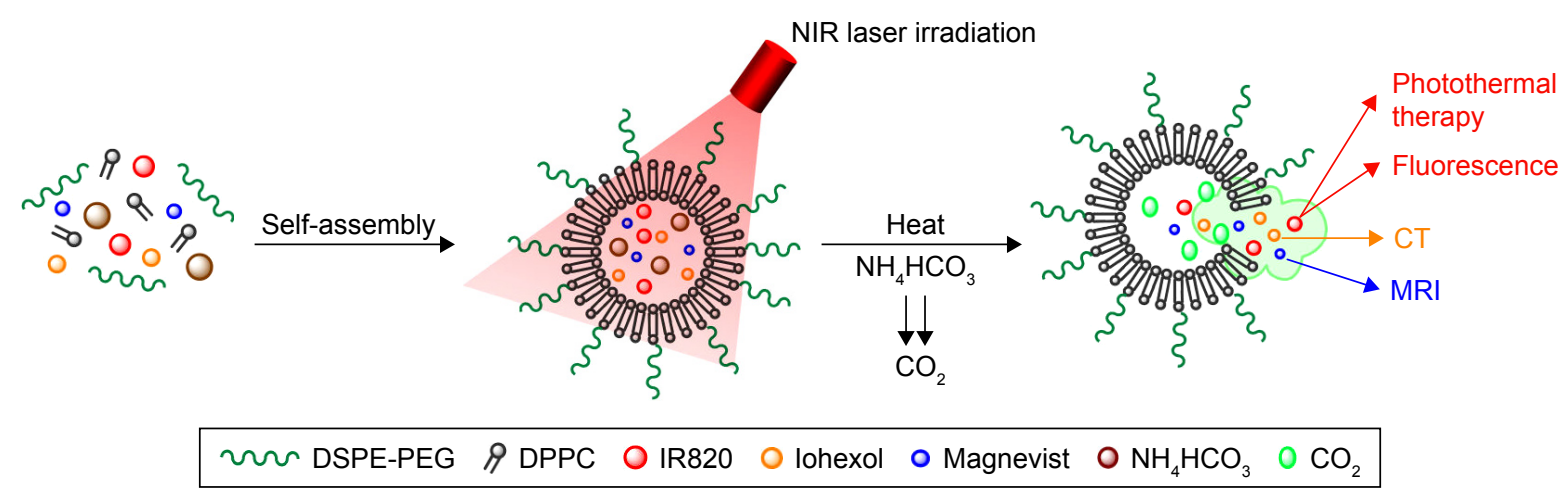

Figure I Schematic illustration of the preparation of LTCAs for CT/MRI/FI imaging and photothermic therapy.

Abbreviations: DSPE-PEG, I,2-distearoyl-sn-glycero-3-phosphoethanolamine- $\mathrm{N}$-[methoxy(polyethylene glycol)]; DPPC, dipalmitoylphosphatidylcholine; FI, fluorescence imaging; LTCAs, light-triggered contrast agents; NIR, near-infrared; CT, computed X-ray tomography; MRI, magnetic resonance imaging.

indicated the fast aggregation and disassembly of LTCAs. As a result of the high conversion efficiency of light into heat of IR820, the laser irradiation gives an impetus to the thermoresponsive phospholipid bilayer (DPPC/DSPC) to reach the phase transition temperature $\left(39^{\circ} \mathrm{C}-42^{\circ} \mathrm{C}\right)$ and to realize responsive release..$^{34}$

\section{In vitro LTCA release}

Encouraged by the light-triggered disassembly performance of LTCAs, the in vitro LTCA release behavior was further investigated using conventional dialysis method with or without NIR irradiation at $1 \mathrm{~W} / \mathrm{cm}^{2}$ for $10 \mathrm{~min}$. The cumulative releases are shown in Figure 3. Due to the phase transition of DPPC/ DSPC by photothermal effect, obviously different release profiles of IR 820 were observed with or without $808 \mathrm{~nm}$ laser irradiation (Figure 3). About $75 \%$ of the payload was released in 60 min after laser irradiation, which showed that IR820 was released effectively from LTCAs after NIR irradiation. By contrast, less than $25 \%$ of IR 820 was released from LTCAs without irradiation (Figure 3). The results indicated that NIR laser irradiation could significantly speed up the release of
CAs from LTCAs, which confirmed the light-triggered release ability of LTCAs. According to previous studies, the phase transition of DPPC and DSPC phospholipid bilayer occurred at a temperature range of $39^{\circ} \mathrm{C}-42^{\circ} \mathrm{C} .{ }^{35,36}$ The lipid bilayer membrane of the LTCAs was broken, and the contents in the core of liposomes were leaked after irradiation. Meanwhile, due to the temperature increase, the generation of $\mathrm{CO}_{2}$ bubbles by disintegration of $\mathrm{NH}_{4} \mathrm{HCO}_{3}$ further accelerated the disruption of LTCAs. ${ }^{37}$ As a result of the phase transition of thermoresponsive phospholipid bilayer and the $\mathrm{CO}_{2}$ generation by $\mathrm{NH}_{4} \mathrm{HCO}_{3}$, the faster light-triggered release might result in high CA concentration gradient to facilitate diffusion of CAs in tumor tissues, which was the key to realize the light-triggered tumor-specific multimodal imaging. ${ }^{38}$

\section{Photothermal profile of LTCAs}

The light-response release profile of IR820 from LTCAs in PBS indicated its potential application in both tumor-specific multimodal imaging and PTT. To confirm the hypothesis, the photothermal effect of LTCAs was also evaluated by monitoring the temperature change under laser irradiation.
A

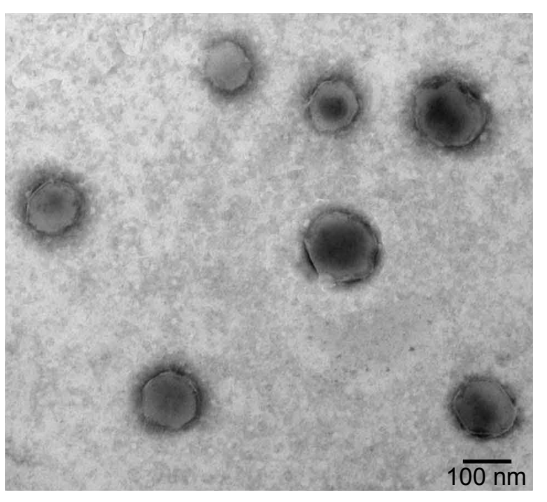

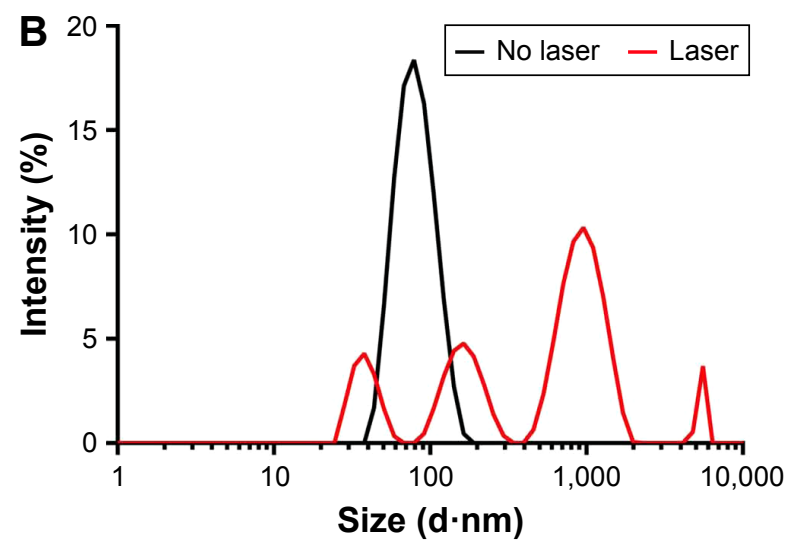

Figure 2 (A) The transmission electron microscopy (TEM) micrographs of LTCAs. (B) The size change of LTCAs before and after irradiation with near-infrared light. Abbreviation: LTCAs, light-triggered contrast agents. 
Table I Physicochemical characterization of LTCAs

\begin{tabular}{llllll}
\hline Samples & Ingredients & Particle size $(\mathbf{n m})$ & Polydispersity index & Encapsulation efficiency (\%) & Loading efficiency (\%) \\
\hline LTCAs & lohexol & & & $15.1 \pm 0.3$ & $3.5 \pm 0.4$ \\
& Magnevist & $113 \pm 8.6$ & $0.11 \pm 0.05$ & $17.6 \pm 0.2$ & $4.1 \pm 0.2$ \\
& IR820 & & $19.8 \pm 0.3$ & $3.2 \pm 0.2$
\end{tabular}

Note: Data represent mean $\pm S D, n=3$.

Abbreviation: LTCA, light-triggered contrast agent.

The maximum temperature of LTCAs increased about $50^{\circ} \mathrm{C}$ with the laser irradiation at $808 \mathrm{~nm}\left(1 \mathrm{~W} / \mathrm{cm}^{2}\right.$ for $\left.10 \mathrm{~min}\right)$, while no obvious temperature increase was observed in the control PBS solution. The photothermal effect was closely related to the concentration of IR820, as shown in Figure 4A. As IR820 concentration increased from 0.5 to $8 \mu \mathrm{M}$, the increased temperatures were improved from $16.7^{\circ} \mathrm{C}$ to $45.5^{\circ} \mathrm{C}$, respectively. In addition, the effect of laser density on the photothermal effect was also evaluated. Under various laser power densities of $0.3,0.5,0.8$, and $1 \mathrm{~W} / \mathrm{cm}^{2}$ for $10 \mathrm{~min}$, the temperature was increased to $32.3^{\circ} \mathrm{C}, 46.9^{\circ} \mathrm{C}$, $71.0^{\circ} \mathrm{C}$, and $77.5^{\circ} \mathrm{C}$, respectively, when LTCA concentration was fixed at $8 \mu \mathrm{M}$ (Figure 4B). These results confirmed the excellent photothermal efficiency of IR820-loaded LTCAs and their potential application in PTT.

\section{PTT in vitro}

It was necessary to determine the cytotoxicity of the LTCAs for further applications in the biomedical field. Cytotoxicity of LTCAs was investigated using standard MTT assay on C6 glioma cancer cells. LTCAs with different concentrations were incubated with $\mathrm{C} 6$ cells for $12 \mathrm{~h}$. As shown in Figure 5A, no obvious cytotoxicity was observed at various concentrations up to $8 \mu \mathrm{M}$ without laser irradiation, which confirmed the low cytotoxicity of LTCAs. However,

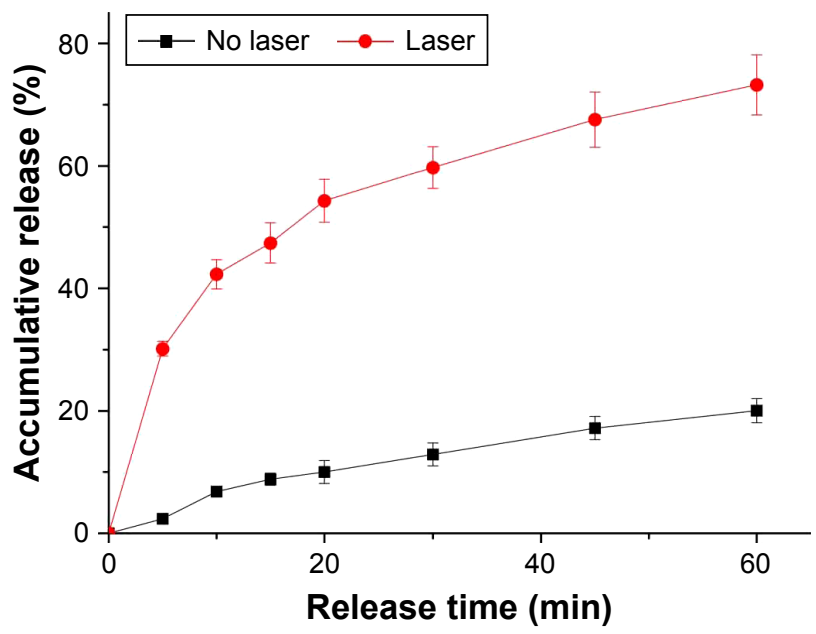

Figure 3 IR820 release profiles from LTCAs with or without near-infrared laser irradiation.

Abbreviation: LTCAs, light-triggered contrast agents. a significant cell inhibiting rate was observed when laser irradiation $\left(1 \mathrm{~W} / \mathrm{cm}^{2}, 15 \mathrm{~min}\right)$ was applied to the C6 cells after incubation with LTCAs. At a concentration of $8 \mu \mathrm{M}$, the relative cell viability decreased to $37 \%$ (Figure 5A). Besides, the high photothermal efficiency was significantly associated with the increased laser power density. The laser irradiation at density of $1.2 \mathrm{~W} / \mathrm{cm}^{2}$ showed the strongest cytotoxicity (79\%) on C6 cells (Figure 5B). As expected, these experiments verified that NIR light-activated heating by IR 820 not only induced CA release from light-sensitive liposome but also improved the PTT effect.

\section{Responsive CT and MRI imaging in vitro}

The major challenge for clinically available CT or MR CAs is the difficulty to distinguish malignant tumors from benign tumors and obtain a high contrast over nearby normal tissues. ${ }^{39}$ Besides, combining different imaging techniques that can fully use the advantages of each while avoiding the drawbacks is a viable option for precision diagnosis since no single modality is perfect and sufficient to obtain all the necessary information. With this consideration, Iohexol and Magnevist, the most widely used CT and MRI (T1)-enhanced CAs in clinic, were both loaded into the LTCA liposome to improve the signal-to-noise ratio of tumor tissues.

To study the effect of light-triggered release on imaging, the CT/MR contrast properties of LTCAs were evaluated in vitro using $\mathrm{C} 6$ cell lines. Firstly, medium containing various concentration of LTCAs after laser irradiation was incubated with $\mathrm{C} 6$ cells for $4 \mathrm{~h}$. After that, cells were collected and scanned by CT/MRI. As shown in Figure 6A, the CT signal intensity improved gradually with the increase of $\mathrm{I}^{-}$concentration $(2,4,6,8$, and $10 \mu \mathrm{M})$. Furthermore, larger Hounsfield unit value was observed in the LTCAs group with laser irradiation, and the difference between the solution with laser and solution without laser at $10 \mu \mathrm{M}$ concentration was 41 Hounsfield units (Figure 6B).

Due to the interaction between Gd(III)-complexes and an inner-sphere water molecule, Gd-complexes could cause the magnetic relaxation of hydrogen nuclei of water, and were used as an important MRI CAs. ${ }^{19}$ Here, before further investigating multimodal imaging of tumor for LTCAs, LTCAs were evaluated for their proton relaxivities $\left(r_{1}\right)$ under 3T MRI systems. 

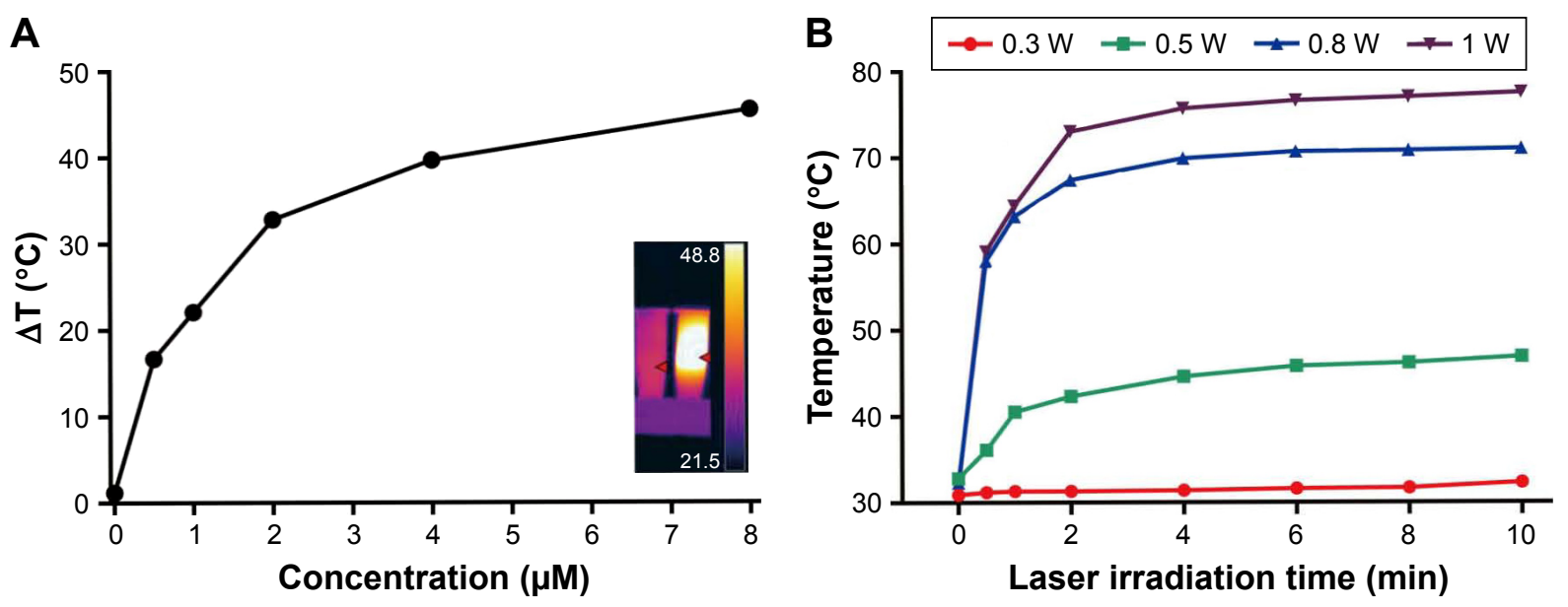

Figure $4(\mathbf{A})$ Plot of temperature change $(\Delta T)$ of LTCAs solution at different concentrations over a period of 10 min laser irradiation $\left(I \mathrm{~W} / \mathrm{cm}^{2}\right)$. The inserted box is the thermal imaging of LTCAs solution after laser irradiation for 10 min. (B) Plot of temperature change of LTCAs solution (IR820: $8 \mu M$ ) with different laser irradiation power. Abbreviation: LTCAs, light-triggered contrast agents.

The T1-weighted (longitudinal relaxation times) MR images of LTCAs were obtained using a heavily T1-weighted fast spoiled gradient echo sequence and measured using different Gd concentrations $(0.06,0.12,0.18,0.24$, and $0.3 \mathrm{mM})$ with or without laser irradiation (Figure 6A). The brightness of MRI of LTCAs was proportional to the increase in Gd concentration. As demonstrated in Figure 6C, LTCAs with laser irradiation shortened the T1 relaxation time. In addition, relaxivity was obtained from the slope of the linear fit of the inverse of $\mathrm{T} 1$ as a function of $\mathrm{Gd}(\mathrm{III})$ concentration. The $\mathrm{r}_{1}$ value of cells incubated with irradiated LTCAs was $7.2 \mathrm{mM}^{-1} \mathrm{~s}^{-1}$, which was approximately 1.79 times higher than that of cells incubated with no irradiation, as shown in Figure 6C. Generally, compared with Gd(III)-entrapped in liposomes, $\mathrm{Gd}(\mathrm{III})$ has proven to show higher ionic relaxivity when Gd(III) was on the surface of liposome. ${ }^{40}$ Due to the release of Gd(III) triggered by laser, more Gd(III) was accumulated in tumor cells and a shortened T1 was observed. These results indicated that the laser irradiation could induce stronger MRI and CT contrast effect in tumors compared to that without irradiation, and suggest that the LTCAs have satisfactory light-triggered response contrast enhancement effect, which indicated the great potential application in responsive $\mathrm{CT} / \mathrm{MRI}$ imaging.

\section{Light-triggered enhancement of MRI and $\mathrm{Fl}$ imaging in vivo}

Currently, CT and MRI are the most powerful imaging techniques used in clinical diagnosis, such as early phase tumor detection and imaging. CT and MRI applications have been becoming more and more dependent on CAs. The multimodal CAs that combine CT, MRI, and FI contrast effect and possess stimulative responsibility may greatly enhance the possibilities to depict the tumor clearly. Nude mice bearing $\mathrm{C} 6$ tumor xenografts were used to investigate the light-triggered imaging capability after intravenous (i.v.) injection of LTCAs. CT images of nude mice with or without laser irradiation are shown in Figure 7A. It can be observed that obvious small patches enhancement at the $1 \mathrm{~h}$ point after
A

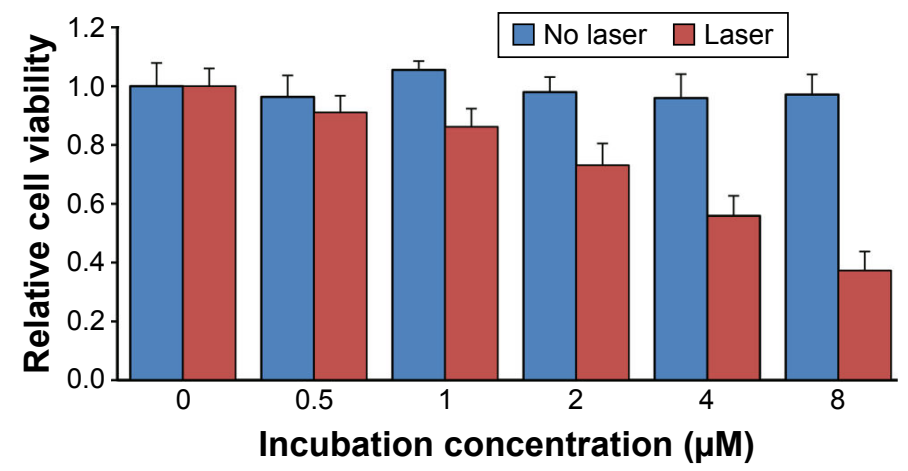

B

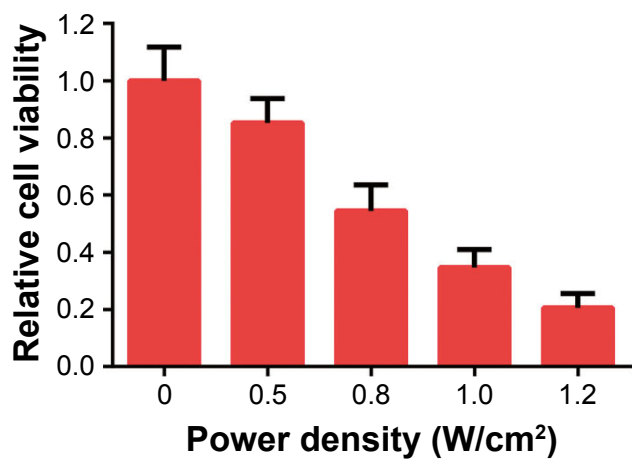

Figure 5 (A) In vitro cytotoxicity of various concentrations LTCAs against C6 cells with or without near-infrared laser irradiation (808 $\mathrm{nm}, \mathrm{I}$ W/ $\mathrm{cm}^{2}$, I5 min). (B) In vitro cytotoxicity of LTCAs (IR820: $8 \mu \mathrm{M}$ ) against C6 cells with different laser irradiation power ( $808 \mathrm{~nm}, 15 \mathrm{~min})$.

Abbreviation: LTCAs, light-triggered contrast agents. 
A
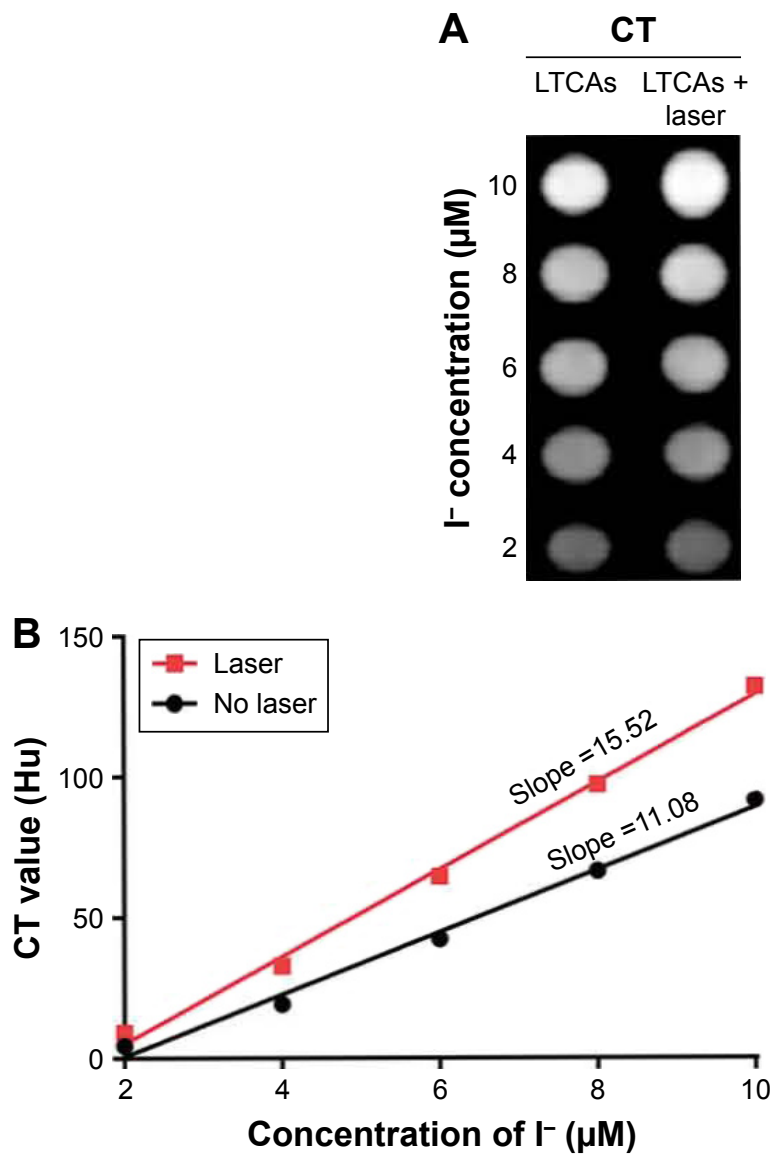
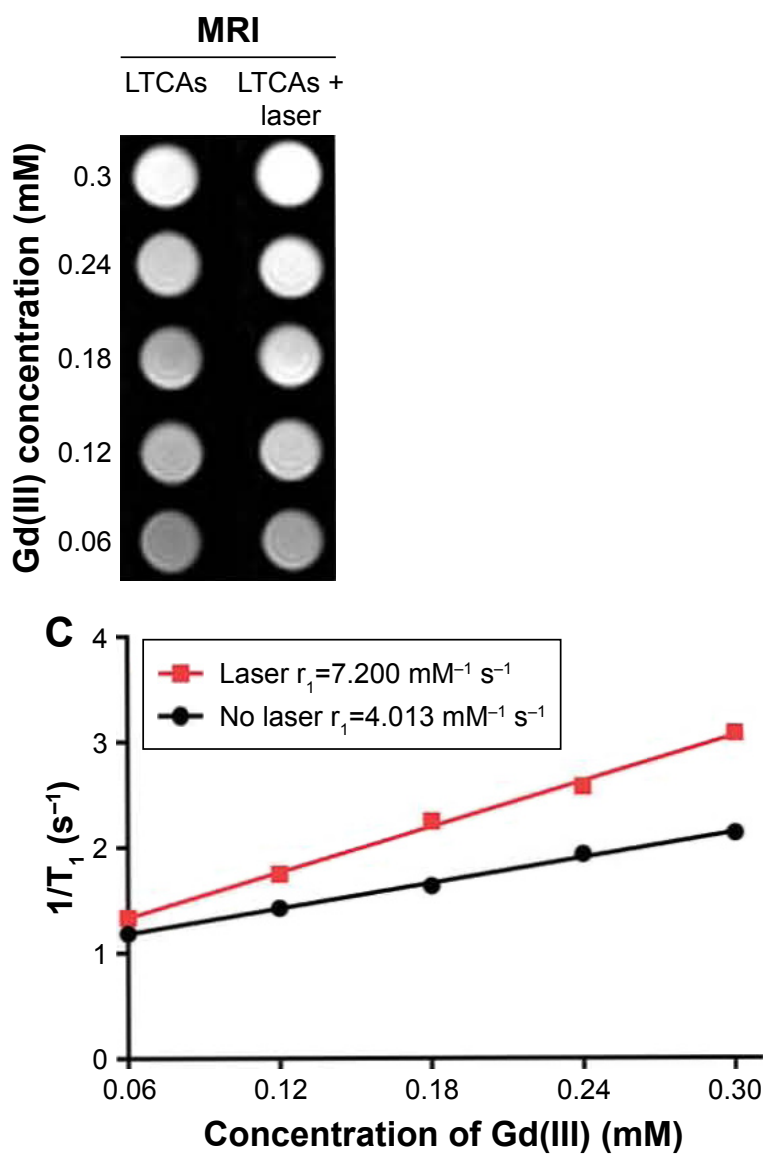

Figure 6 (A) In vitro CT values and TI-weighted images with or without near-infrared laser irradiation at different concentrations. A plot of CT values (B) and relative relaxation rate $(\mathbf{C})$ with or without near-infrared laser irradiation at different $\mathrm{I}^{-}(\mathbf{B})$ and $\mathrm{Gd}(\mathrm{III})$ (C) concentrations.

Abbreviations: CT, computed X-ray tomography; LTCA, light-triggered contrast agent; MRI, magnetic resonance imaging.

LTCAs injection with or without laser irradiation. Moreover, the whole tumor became much brighter at $6 \mathrm{~h}$. Enhancement percentage with laser irradiation as indicated by accurate Hounsfield unit value was $32 \%$ compared to $29 \%$ without laser irradiation (Figure 7B).

For MRI evaluation, due to the faster release of Gd(III) at the tumor site by light-induced membrane rupture, a higher cumulated concentration of Gd(III) was observed in tumor cells. The cumulative concentration of Magnevist has an appreciable effect on the MRI contrast enhancement. As a result, T1-weighted images were much brighter after i.v. injection of LTCAs with irradiation, than without irradiation, at different time points (Figure 7C), and the relative $\mathrm{T} 1$ signal intensity in mice with irradiation increased by $47 \%$, while the intensity was $17 \%$ in mice without irradiation (Figure 7D). These multifunctional LTCAs benefit the multimodal imaging tumor detection and real-time guided PTT treatment with minimal damage to normal tissues. ${ }^{12-14}$ The result indicated tumor-specific MRI imaging enhancement ability and the potential application of LTCAs.

As for FI, an in vivo fluorescence system was employed to observe the emission fluorescence using nude mice bearing
C6 tumor xenografts. As the fluorescence was self-quenched under high concentrations, ${ }^{41}$ IR 820 showed weak fluorescence intensity before laser irradiation when it was encapsulated in the core of the LTCAs. After laser irradiation, IR820 was quickly released from LTCAs, and stronger fluorescence intensity appeared at the tumor region and gradually increased at the time points 1,2 , and $6 \mathrm{~h}$ with the laser irradiation (Figure 8). Nevertheless, no obvious fluorescence signal changes were observed in the absence of laser irradiation (Figure 8). The remarkable FI enhancement after i.v. injection with laser irradiation was mainly induced by responsive release and accumulation of IR820 at the tumor site.

\section{In vivo photothermal treatment}

For the evaluation of the PTT effect, nude mice bearing C6 tumor xenografts were treated by intravenously injecting LTCAs or PBS, and then were exposed to NIR laser $\left(1 \mathrm{~W} / \mathrm{cm}^{2}\right.$, $20 \mathrm{~min}$ ). Infrared thermal imaging system was employed to record the temperature of the tumor area (Figure 9A). Under NIR irradiation, the tumor surface temperature of the LTCAs + laser group rapidly increased from $30.8^{\circ} \mathrm{C}$ to $55.1^{\circ} \mathrm{C}$ (Figure 9B). Given that quickly increased temperature 

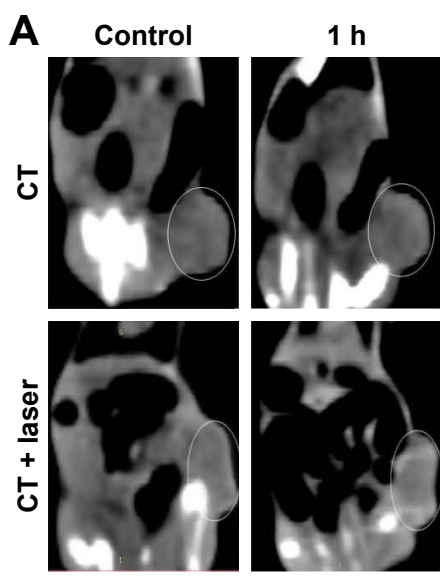

C Control
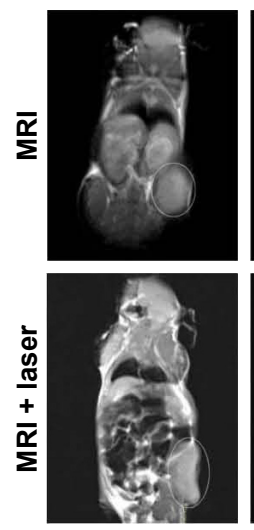

$1 \mathrm{~h}$
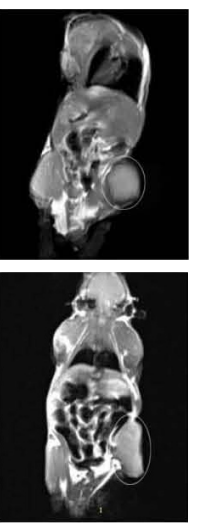

$2 \mathrm{~h}$
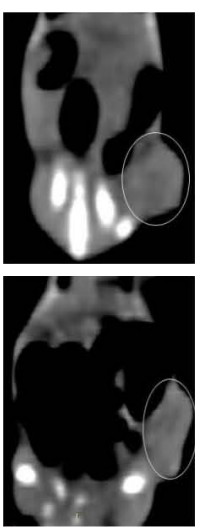

$2 \mathrm{~h}$
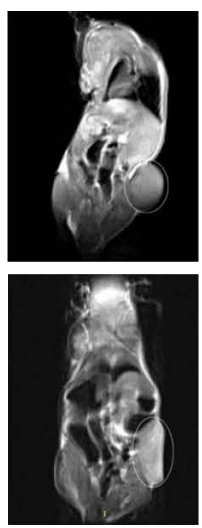
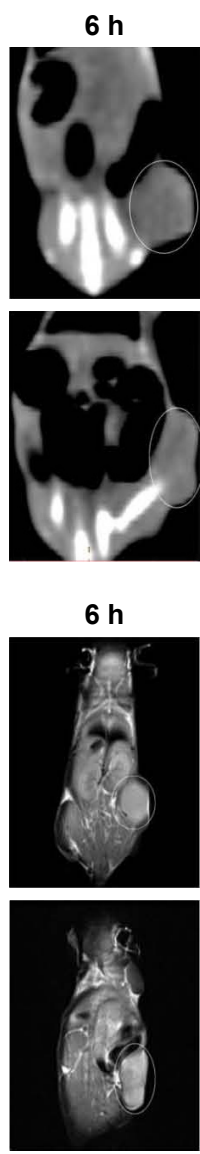

B

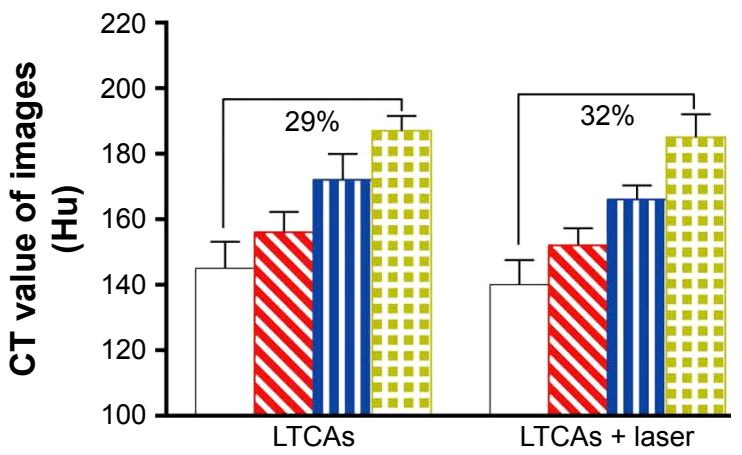

D

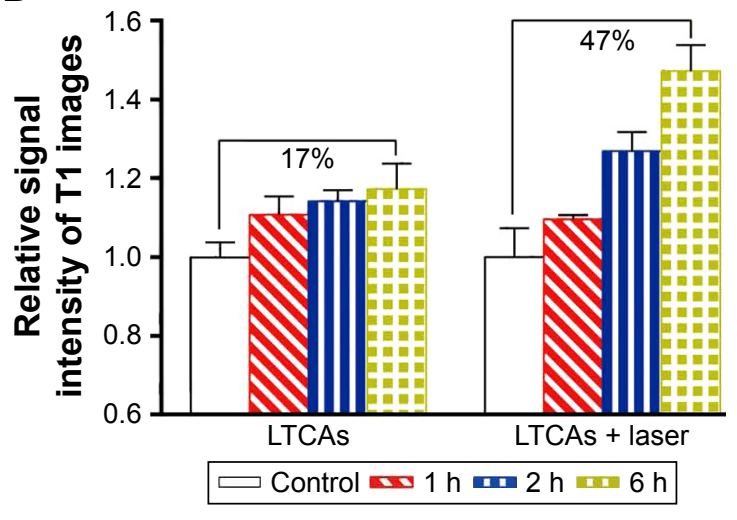

Figure 7 In vivo CT imaging (A) and contrast enhancement values (B) in C6 tumor-bearing Balb/c nude mice before and post i.v. injection LTCAs at different time intervals with or without near-infrared laser irradiation. In vivo MRI imaging (C) and relative signal intensity (D) in C6 tumor-bearing Balb/c nude mice before and post i.v. injection LTCAs at different time intervals with or without near-infrared laser irradiation.

Abbreviations: CT, computed x-ray tomography; i.v., intravenous; LTCA, light-triggered contrast agent; MRI, magnetic resonance imaging.
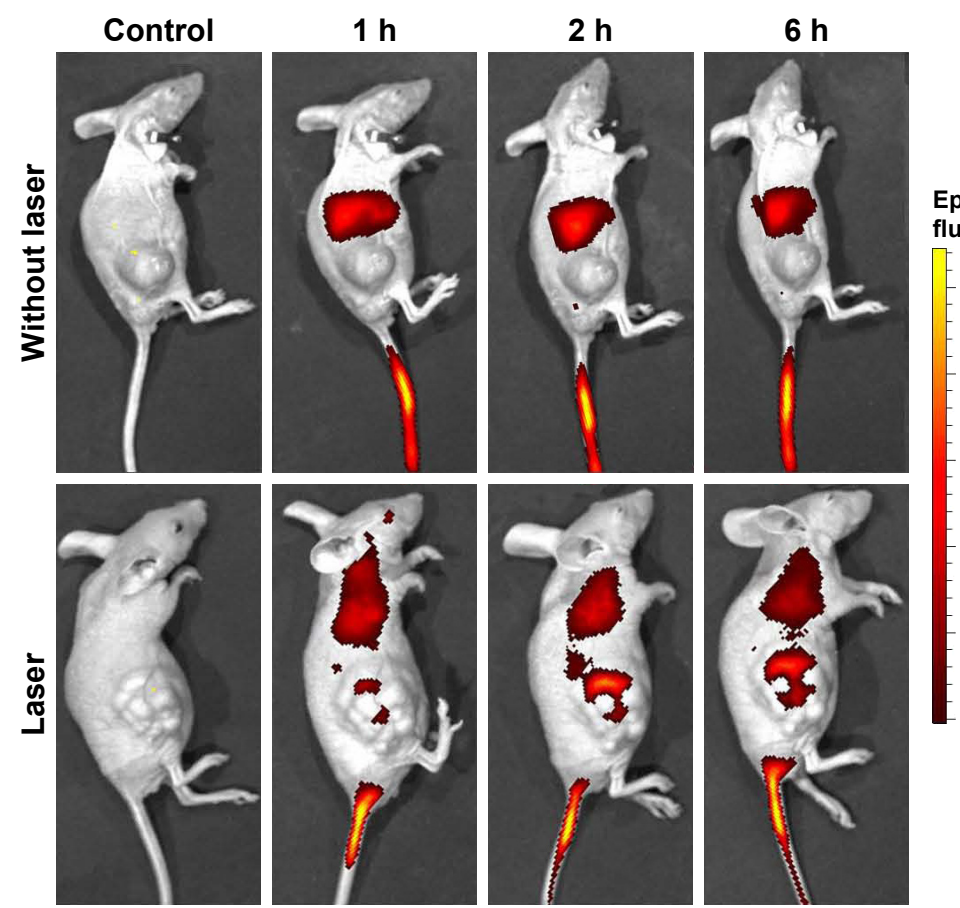

fluorescence

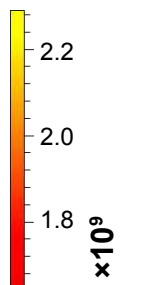

Figure 8 In vivo fluorescence imaging in C6 tumor-bearing Balb/c nude mice before and post i.v. injection LTCAs at different time intervals with or without near-infrared laser irradiation.

Abbreviations: i.v., intravenous; LTCA, light-triggered contrast agent. 

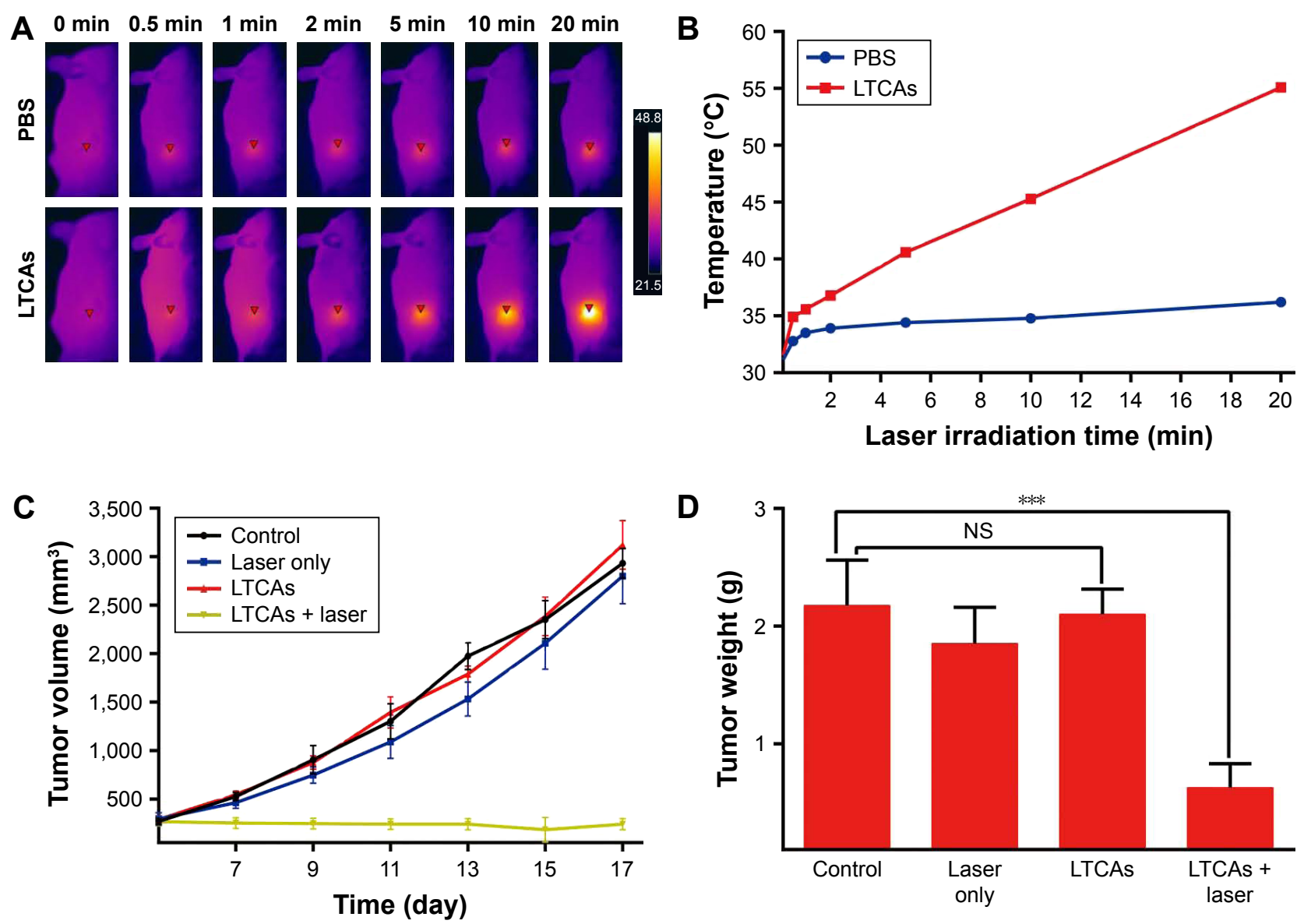

E

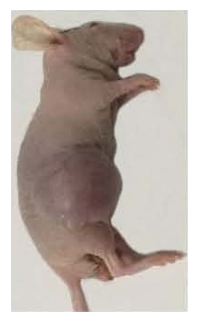

Control

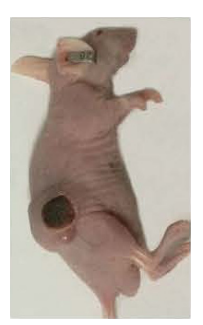

Laser only

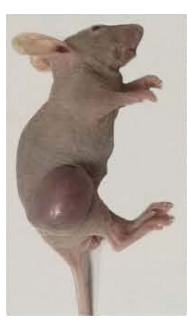

LTCAs

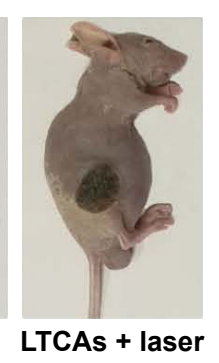

LTCAs + laser
$\mathbf{F}$

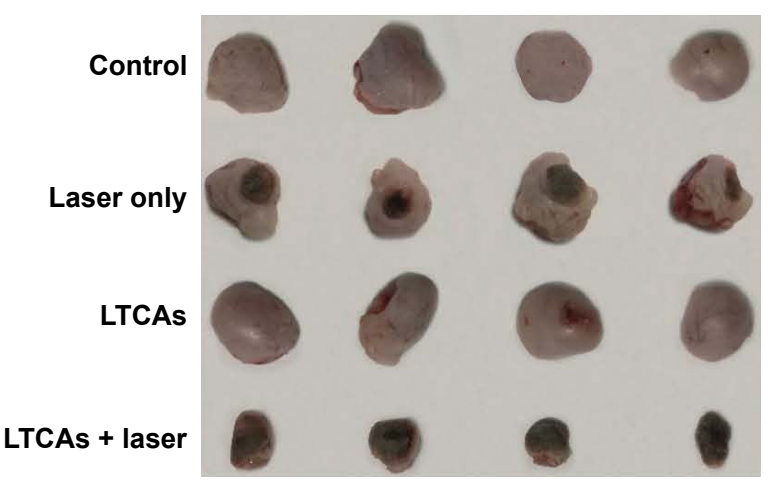

LTCAs

LTCAs + laser

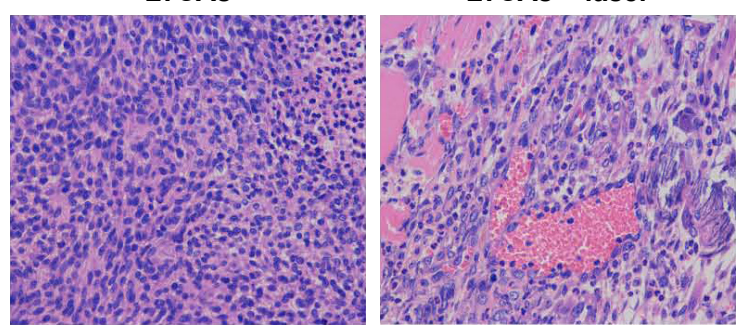

Figure 9 (A) In vivo near-infrared laser irradiation $(808 \mathrm{~nm}, \mathrm{I}$ W/cm²) photothermal images of C6 tumor-bearing Balb/c nude mice with i.v. injection of PBS or LTCAs $(200 \mu \mathrm{L})$ taken at different time intervals. (B) The photothermal profiles of PBS or LTCAs injected mice at different time intervals under $808 \mathrm{~nm}$ laser irradiation with a power

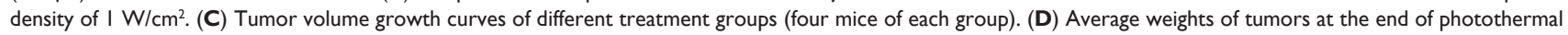
treatment. $P$-values: $* * *(P<0.001)$, NS (no significant difference, $P>0.05$ ), calculated by analysis of variance (ANOVA). (E) Photos of $C 6$ tumor-bearing Balb/c nude mice taken 17 days after different treatments. (F) Tumor photos from different groups of $\mathrm{C} 6$ tumor-bearing Balb/c nude mice at the end of treatment. (G) hematoxylin-eosin staining photos of collected tumors from different groups.

Abbreviations: i.v., intravenous; LTCA, light-triggered contrast agent; NS, not significant. 
over $42.5^{\circ} \mathrm{C}$ could lead to the death of cancer cells, ${ }^{42-44}$ the result verified the excellent light-induced hyperthermia and PTT effect of LTCAs. By contrast, no obvious temperature increase at the tumor site was recorded in the control group and surrounding normal tissues in LATCAs group.

To further investigate the PTT performance of LTCAs, the mice were divided into four groups when the tumor volume grew to $200 \mathrm{~mm}^{3}$ on average. The tumor volumes of the four groups were measured for 17 days as shown in Figure 9C. It can be seen that the PBS-treated group showed rapid tumor growth (Figure 9C) with an average tumor volume above $2.9 \times 10^{3} \mathrm{~mm}^{3}$ at the end of the experiment. Treatment with LTCAs + laser showed efficient tumor inhibition and complete eradication in the treatment period. The tumor growth was suppressed up to $8 \%$ compared to PBS control mice, which indicated excellent tumor growth inhibition of LTCAs with laser irradiation. By contrast, the mice irradiated by laser only or injected only LTCAs showed slight suppression of tumor growth.

The tumor photographs and average weights of each group were also recorded, which are shown in Figure 9D-F. The group of LTCAs + laser had the lightest tumor weight among the four groups. H-E staining showed that the LTCAs + laser group had more apoptotic tissues and nuclear fragmentation (Figure 9G), which are consistent with the tumor biology behavior. These results suggested the efficient PTT effect of LTCAs and their potential application in cancer therapy.

\section{Conclusion}

In summary, LTCAs have been successfully prepared for light-triggered enhancement of CT/MRI/FI trimodal imaging and imaging-guided tumor PTT therapy. Both tumor-specific multimodal imaging activated by lighttriggered release of CAs and local hyperthermia generated by IR820 under NIR irradiation were integrated into the novel liposome formulation of LTCAs, which provided selective and local imaging and destruction of tumor with the aid of NIR laser irradiation. As expected, the nanoparticles released CAs after NIR laser irradiation and boosted their relaxivity or intensity selectively, which proved the feasibility and validity of this strategy in resourceful and selective tumor imaging. Additionally, due to the high conversion efficiency of light into heat of IR820, LTCA as a PTT agent was demonstrated to possess ideal photothermal effect and satisfactory antitumor ability after NIR laser irradiation. The results indicate that LTCAs hold significant promise in the CA platform for tumor-specific enhanced multimodal imaging and PTT agents.

\section{Acknowledgments}

This work was financially supported by National Natural Science Foundation of China (81372369, 51473127, 8157 1734, 51673152) and Opening Project of Key Laboratory of Biomedical Polymers of Ministry of Education at Wuhan University (20150103).

\section{Disclosure}

The authors report no conflicts of interest in this work.

\section{References}

1. Haug AR, Cindea-Drimus R, Auernhammer CJ, et al. Neuroendocrine tumor recurrence: diagnosis with 68Ga-DOTATATE PET/CT. Radiology. 2014;270(2):517-525.

2. Liu Y, Ashton JR, Moding EJ, et al. A plasmonic gold nanostar theranostic probe for in vivo tumor imaging and photothermal therapy. Theranostics. 2015;5(9):946-960.

3. Hainfeld JF, Slatkin DN, Focella TM, Smilowitz HM. Gold nanoparticles: a new X-ray contrast agent. Br J Radiol. 2006;79(939):248-253.

4. Floyd WC 3rd, Klemm PJ, Smiles DE, et al. Conjugation effects of various linkers on Gd(III) MRI contrast agents with dendrimers: optimizing the hydroxypyridinonate (HOPO) ligands with nontoxic, degradable esteramide (EA) dendrimers for high relaxivity. $J$ Am Chem Soc. 2011;133(8):2390-2393.

5. Chen Y, Yin Q, Ji X, et al. Manganese oxide-based multifunctionalized mesoporous silica nanoparticles for $\mathrm{pH}$-responsive MRI, ultrasonography and circumvention of MDR in cancer cells. Biomaterials. 2012;33(29):7126-7137.

6. Kim T, Cho EJ, Chae Y, et al. Urchin-shaped manganese oxide nanoparticles as pH-responsive activatable $\mathrm{T} 1$ contrast agents for magnetic resonance imaging. Angew Chem Int Ed Engl. 2011;50(45):10589-10593.

7. Gao GH, Im GH, Kim MS, et al. Magnetite-nanoparticle-encapsulated pH-responsive polymeric micelle as an MRI probe for detecting acidic pathologic areas. Small. 2010;6(11):1201-1204.

8. Sowers MA, McCombs JR, Wang Y, et al. Redox-responsive branchedbottlebrush polymers for in vivo MRI and fluorescence imaging. Nat Commun. 2014;5:5460.

9. de Smet M, Heijman E, Langereis S, Hijnen NM, Grüll H. Magnetic resonance imaging of high intensity focused ultrasound mediated drug delivery from temperature-sensitive liposomes: an in vivo proof-ofconcept study. J Control Release. 2011;150(1):102-110.

10. Opoku-Damoah Y, Wang R, Zhou J, Ding Y. Versatile nanosystembased cancer theranostics: design inspiration and predetermined routing. Theranostics. 2016;6(7):986-1003.

11. Jayapaul J, Arns S, Bunker M, et al. In vivo evaluation of riboflavin receptor targeted fluorescent USPIO in mice with prostate cancer xenografts. Nano Res. 2016;9(5):1319-1333.

12. Li M, Teh C, Ang CY, et al. Near-infrared light-absorptive stealth liposomes for localized photothermal ablation of tumors combined with chemotherapy. Adv Funct Mater. 2015;25(35):5602-5610.

13. Yang X, Liu X, Liu Z, Pu F, Ren J, Qu X. Near-infrared light-triggered, targeted drug delivery to cancer cells by aptamer gated nanovehicles. Adv Mater. 2012;24(21):2890-2895.

14. Chen Q, Liang C, Wang X, He J, Li Y, Liu Z. An albumin-based theranostic nano-agent for dual-modal imaging guided photothermal therapy to inhibit lymphatic metastasis of cancer post surgery. Biomaterials. 2014;35(34):9355-9362.

15. Liang Y, Gao W, Peng X, et al. Near infrared light responsive hybrid nanoparticles for synergistic therapy. Biomaterials. 2016;100: 76-90.

16. Liu J, Zheng X, Yan L, et al. Bismuth sulfide nanorods as a precision nanomedicine for in vivo multimodal imaging-guided photothermal therapy of tumor. ACS Nano. 2015;9(1):696-707. 
17. Qiu X, Xu L, Zhang Y, et al. Photothermal ablation of in situ renal tumor by PEG-IR780-C13 micelles and near-infrared irradiation. Mol Pharm. 2016;13(3):829-838.

18. Song J, Huang P, Duan H, Chen X. Plasmonic vesicles of amphiphilic nanocrystals: optically active multifunctional platform for cancer diagnosis and therapy. Acc Chem Res. 2015;48(9):2506-2515.

19. Jung SH, Na K, Lee SA, Cho SH, Seong H, Shin BC. Gd(III)-DOTAmodified sonosensitive liposomes for ultrasound-triggered release and MR imaging. Nanoscale Res Lett. 2012;7(1):462.

20. Yang K, Hu L, Ma X, et al. Multimodal imaging guided photothermal therapy using functionalized graphene nanosheets anchored with magnetic nanoparticles. Adv Mat. 2012;24(14):1868-1872.

21. Cheng L, Liu J, Gu X, et al. PEGylated WS(2) nanosheets as a multifunctional theranostic agent for in vivo dual-modal $\mathrm{CT} /$ photoacoustic imaging guided photothermal therapy. Adv Mater. 2014;26(12): 1886-1893.

22. Zhang L, Gao S, Zhang F, Yang K, Ma Q, Zhu L. Activatable hyaluronic acid nanoparticle as a theranostic agent for optical/photoacoustic imageguided photothermal therapy. ACS Nano. 2014;8(12):12250-12258.

23. Johnston J, Taylor EN, Gilbert RJ, Webster TJ. Improved molecular fingerprint analysis employing multi-branched gold nanoparticles in conjunction with surface-enhanced Raman scattering. Int J Nanomedicine. 2016;11:45-53.

24. Srinivasan S, Manchanda R, Fernandez-Fernandez A, Lei T, McGoron AJ. Near-infrared fluorescing IR820-chitosan conjugate for multifunctional cancer theranostic applications. J Photochem Photobiol B Biol. 2013; 119:52-59.

25. Qian J, Wang D, Cai F, Zhan Q, Wang Y, He S. Photosensitizer encapsulated organically modified silica nanoparticles for direct two-photon photodynamic therapy and in vivo functional imaging. Biomaterials. 2012;33(19):4851-4860.

26. Peng J, Qi T, Liao J, et al. Mesoporous magnetic gold "nanoclusters" as theranostic carrier for chemo-photothermal co-therapy of breast cancer. Theranostics. 2014;4(7):678-692.

27. Li W, Peng J, Tan L, et al. Mild photothermal therapy/photodynamic therapy/chemotherapy of breast cancer by Lyp-1 modified Docetaxel/ IR820 Co-loaded micelles. Biomaterials. 2016;106:119-133.

28. Wu B, Yu P, Cui C, et al. Folate-containing reduction-sensitive lipidpolymer hybrid nanoparticles for targeted delivery of doxorubicin. Biomater Sci. 2015;3(4):655-664.

29. Na K, Lee SA, Jung SH, Shin BC. Gadolinium-based cancer therapeutic liposomes for chemotherapeutics and diagnostics. Colloids Surf B Biointerfaces. 2011;84(1):82-87.

30. Cittadino E, Ferraretto M, Torres E, et al. MRI evaluation of the antitumor activity of paramagnetic liposomes loaded with prednisolone phosphate. Eur J Pharm Sci. 2012;45(4):436-441.
31. Yan GP, Robinson L, Hogg P. Magnetic resonance imaging contrast agents: overview and perspectives. Radiography. 2007;13(Suppl 1): e5-e19.

32. Zhang D, Wu M, Zeng Y, et al. Lipid micelles packaged with semiconducting polymer dots as simultaneous MRI/photoacoustic imaging and photodynamic/photothermal dual-modal therapeutic agents for liver cancer. J Mater Chem B. 2016;4(4):589-599.

33. Zak B, Willard HH, Myers GB, Boyle AJ. Chloric acid method for determination of protein-bound iodine. Anal Chem. 1952;24(8):1345-1348.

34. Dicheva BM, ten Hagen TL, Schipper D, et al. Targeted and heattriggered doxorubicin delivery to tumors by dual targeted cationic thermosensitive liposomes. J Control Release. 2014;195:37-48.

35. Koning GA, Li L, ten Hagen TL. Thermosensitive liposomes for the delivery of cancer therapeutics. Ther Deliv. 2010;1(5):707-711.

36. Wang F, Liu J. Liposome supported metal oxide nanoparticles: interaction mechanism, light controlled content release, and intracellular delivery. Small. 2014;10(19):3927-3931.

37. Chung MF, Chen KJ, Liang HF, et al. A liposomal system capable of generating $\mathrm{CO} 2$ bubbles to induce transient cavitation, lysosomal rupturing, and cell necrosis. Angew Chem Int Ed Engl. 2012;51(40): 10089-10093.

38. Guo F, Yu M, Wang J, Tan F, Li N. Smart IR780 theranostic nanocarrier for tumor-specific therapy: hyperthermia-mediated bubblegenerating and folate-targeted liposomes. ACS Appl Mater Interfaces. 2015;7(37):20556-20567.

39. Zhang Y, Cao Y, Shih GL, Hecht EM, Prince MR. Extent of signal hyperintensity on unenhanced T1-weighted brain MR images after more than 35 administrations of linear gadolinium-based contrast agents. Radiology. 2016;282(2):516-525

40. Accardo A, Tesauro D, Aloj L, Pedone C, Morelli G. Supramolecular aggregates containing lipophilic Gd(III) complexes as contrast agents in MRI. Coord Chem Rev. 2009;253(17-18):2193-2213.

41. Xie C, Upputuri PK, Zhen X, Pramanik M, Pu K. Self-quenched semiconducting polymer nanoparticles for amplified in vivo photoacoustic imaging. Biomaterials. 2017;119:1-8.

42. Dewey WC, Hopwood LE, Sapareto SA, Gerweck LE. Cellular responses to combinations of hyperthermia and radiation. Radiology. 1977;123(2):463-474.

43. Yue C, Liu P, Zheng M, et al. IR-780 dye loaded tumor targeting theranostic nanoparticles for NIR imaging and photothermal therapy. Biomaterials. 2013;34(28):6853-6861.

44. Tian Q, Jiang F, Zou R, et al. Hydrophilic Cu9S5 nanocrystals: a photothermal agent with a $25.7 \%$ heat conversion efficiency for photothermal ablation of cancer cells in vivo. ACS Nano. 2011;5(12):9761-9771.
International Journal of Nanomedicine

\section{Publish your work in this journal}

The International Journal of Nanomedicine is an international, peerreviewed journal focusing on the application of nanotechnology in diagnostics, therapeutics, and drug delivery systems throughout the biomedical field. This journal is indexed on PubMed Central, MedLine, CAS, SciSearch ${ }^{\circledR}$, Current Contents ${ }^{\circledR} /$ Clinical Medicine,
Dovepress

Journal Citation Reports/Science Edition, EMBase, Scopus and the Elsevier Bibliographic databases. The manuscript management system is completely online and includes a very quick and fair peer-review system, which is all easy to use. Visit http://www.dovepress.com/ testimonials.php to read real quotes from published authors. 